\title{
Erratum
}

\section{The Spectral Class of the Quantum-Mechanical Oscillator}

\author{
H. P. McKean ${ }^{1}$ and E. Trubowitz ${ }^{2}$ \\ 1 Courant Institute of Mathematical Sciences, New York University, New York, NY 10012, \\ USA \\ 2 ETH, CH-8093 Zürich, Switzerland
}

B. Levitan (Moscow State Univ.) has kindly pointed out two places at which the proofs are inadequate. The first occurs on p. 481 where it is stated that the contribution to $\int e_{n} f_{n}^{0} \Delta q d x$ from $|x| \geqq n^{-1 / 6-}$ is rapidly vanishing. The estimates advanced do not support this, but $\mathrm{B}$. Levitan says that $\left\|e_{n} f_{n}^{0}\right\|_{2} \leqq n^{-1 / 2+}(n \uparrow \infty)$, which suffices in view of the rapid vanishing of $\Delta q$. The proof is reported to be complicated. The second point occurs on $p .482$ where the vanishing of the relative trace $H=\int\left[p(t, x, x)-p^{0}(t, x, x)\right] d x$ is said to prove the vanishing of the relative KDV invariants $J_{n}: n \geqq 0$ via the expansion $H=(4 \pi t)^{-1 / 2}\left(J_{0} t+J_{1} t^{2}+\ldots\right)(t \downarrow 0)$. The idea is this. Let $Q=\int_{0}^{t} q[x(s)] d s$, in which $x(t): t \geqq 0$ is the standard Brownian motion with infinitesimal operator $\partial^{2} / \partial x^{2}$. Then

$$
\sqrt{4 \pi t} H=\int E_{x}\left[e^{-Q}-e^{-Q^{0}} \mid x(t)=x\right] d x=\sum_{n=1}^{\infty} \frac{(-1)^{n}}{n !} \int E_{x}\left[(\Delta Q)^{n} e^{-Q^{0}} \mid x(t)=x\right] d x .
$$

The expression is exact, producing an error $\leqq$ constant $\times t^{n}$ upon breaking it off after $n-1$ terms. The individual terms are now developed in powers of $t$. The computation is routine, the rapid vanishing of $\Delta q$ providing the necessary domination to the formal manipulations.

Communicated by A. Jaffe

Received April 5, 1985 